\title{
ULTRASONOGRAPHY OF THE OPTIC DISC CUP IN DISCS OF VARIOUS SIZES
}

\author{
S. WINDER and H. R. ATTA \\ Aberdeen
}

\begin{abstract}
SUMMARY
Purpose: The aim of the study was to evaluate the sensitivity of modern, eye-dedicated B-scanners in detecting optic disc cups and to correlate the echographic measurements with an optical assessment. A secondary aim was to correlate the rim-to-rim diameter of the optic disc and the width of the retrobulbar optic nerve on standardised $A$-scan.

Methods: A prospective study was conducted on 25 emmetropic subjects (50 eyes) with discs and cups of various sizes. They were examined optically by one observer using a high-magnification slit lamp and the 90 D Volk lens. Another observer measured the B-scan dimensions of the disc cups and the retrobulbar optic nerve. Data from the two observers were compared at the end of the study.

Results: High resolution B-scan detected optic disc cups of $0.5 \mathrm{~mm}$ or larger in all cases. A statistically significant correlation was obtained between echographic measurements and both vertical optical cup disc diameters and cup disc $(\mathrm{C}: \mathrm{D})$ ratios $(p<0.0001)$. Large C:D ratios (0.6 or larger) but small rim-to-rim disc diameter were reported as 'small' on echography. No correlation was found between the measurement of retrobulbar nerve and the diameter of the vertical optical disc diameter.

Conclusion: Modern, eye-dedicated ultrasonographic scanners are capable of detecting optic disc cups of 0.5 $\mathrm{mm}$ or larger. As there is a normal variation in optic disc rim-to-rim diameter, and this cannot be measured using ultrasound, we suggest the reporting of a $C: D$ ratio on ultrasonography to be potentially misleading. Large disc cups are, however, easily seen and many are likely to be 'pathological'.
\end{abstract}

Ultrasonography is a helpful tool in the diagnosis of many ocular abnormalities, particularly when ophthalmoscopy is hampered by media opacities. Such abnormalities include subtle lesions such as macular oedema and optic disc cupping. The desire

Correspondence to: Mr S. Winder, Eye Clinic, Aberdeen Royal Infirmary, Foresterhill, Aberdeen AB9 2ZB, UK. to identify these changes is evident, for example, during echographic screening of patients with dense cataract.

Few studies have attempted to test the ability of modern B-scanners to detect optic disc cupping. ${ }^{1-4}$ In these studies correlation was made between the ultrasonographic cup measurements and the cup disc $(C: D)$ ratio. As there is a normal variation in optic disc diameter ${ }^{5-8}$ and the B-scanner is only able to detect the disc cup, we suggest that quoting a ratio may be potentially misleading to the clinician. We therefore undertook a study of patients with differing cup sizes and disc sizes with the primary aims of (1) determining the ability of modern eye-dedicated Bscanners to detect disc cups, and (2) correlating the optical (vertical) diameter of the cup and $\mathrm{C}: \mathrm{D}$ ratio on the one hand and the echographic appearance of the cups on the other. A secondary aim was to determine whether there is a correlation between 'rim-to-rim' diameter of the optic disc and the width of the retrobulbar optic nerve, as measured with standardised A-scan.

\section{METHODS}

We examined 25 patients who were selected by the first author (S.W.). Subjects were recruited from the general clinics in the outpatient department. Entry criteria were that the patient had clear media and was within 2 dioptres of emmetropia. After an explanation of the study and the safety of ultrasound, informed consent was obtained. Patients who were unwilling to take part, those with active ocular surface disease, anterior segment disease or recent ocular surgery were excluded.

\section{Optical Examination}

Patients were examined by author 1 after dilatation of the pupil. Optic disc vertical diameter was measured using a calibrated Haag Streit slit lamp and a Volk $90 \mathrm{D}$ lens. At maximum magnification the vertical slit height was adjusted to the vertical height 


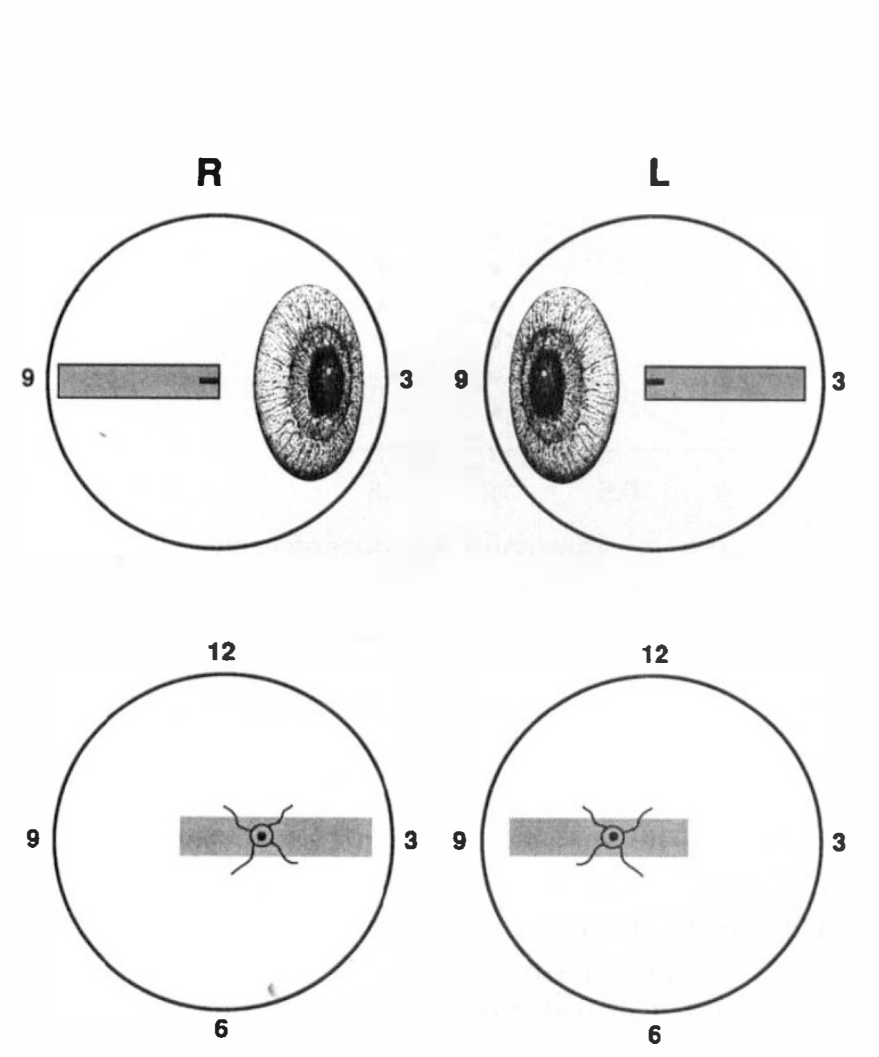

(a)
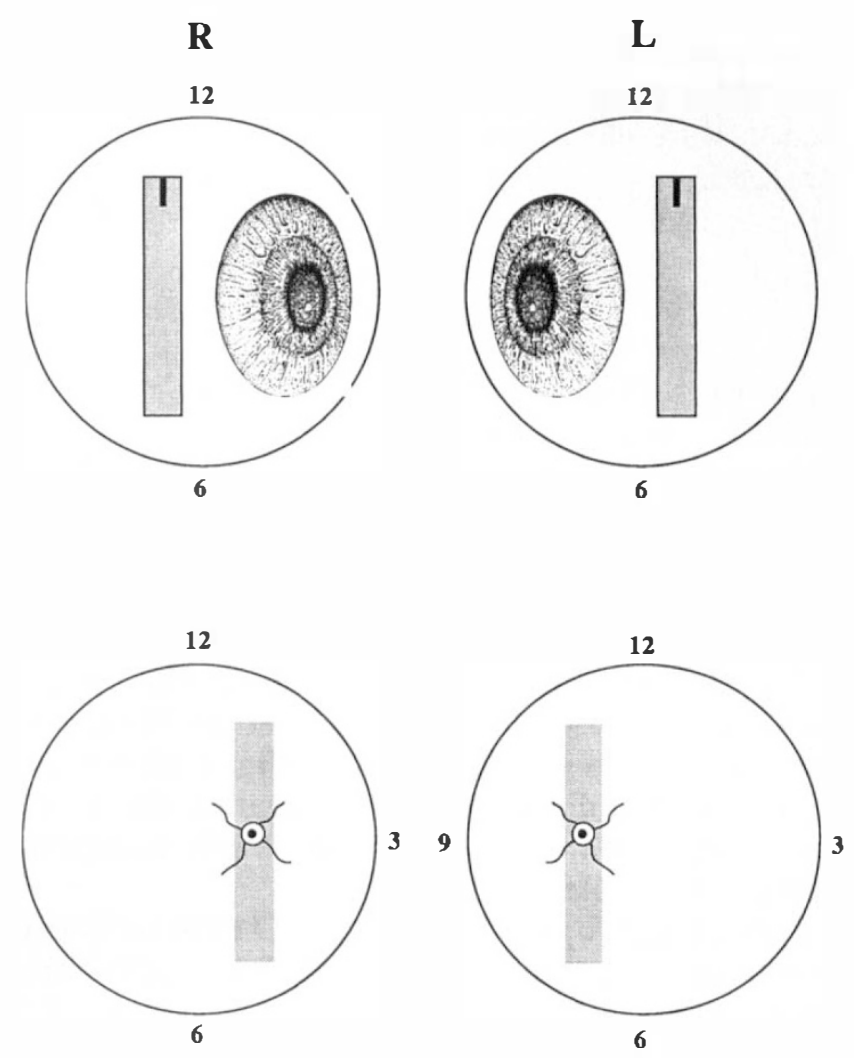

(b)

Fig. 1. The position of the ultrasound probe on the globe to obtain the transverse (a) and longitudinal (b) sections used in our study, with a to-sclera approach.

of the disc and the reading of the measurement then taken from the scale. The $\mathrm{C}: \mathrm{D}$ ratio was estimated clinically and then the slit turned to $90^{\circ}$ and the horizontal scale used to measure the difference between the cup and diameter; the ratio was then calculated. This group included patients with various $\mathrm{C}: \mathrm{D}$ ratios and patients with small disc diameters. The figures were converted using a recently published paper. ${ }^{9}$ After this an ultrasound appointment was made for the patient.

\section{Echographic Measurement}

Ultrasonography was performed by author 2 (H.R.A.), who was unaware of the findings of author 1. The ultrasound instruments used were the Ophthascan-s and Ophthascan Mini-A (Alcon Laboratories, UK). The first provides a high-resolution contact B-scan with eye-dedicated transducer and freeze frame facility. The latter is a 'standardised' A-scan, also with freeze frame facility and electronic callipers.

B-scan examinations were carried out using a medium (60-70 dB) gain setting to optimise resolution of the optic nerve head. The grey scale was also reduced to improve contrast and produce welldefined margins of the disc cup whenever possible. The decibel gain and grey scale were not standard- ised for all patients, as each examination required slightly different settings for optimal display of the optic disc cup (standardisation would have been a prerequisite if temporal observations were part of the study). Scanning was conducted on the anaesthetised globe, directly on the sclera to enhance the quality of images. Transverse (cross-section) and longitudinal (long-section) scans were performed on both optic nerve heads giving vertical and horizontal sections of the disc cup (Fig. 1). Both sections have the advantage of avoiding the lens and allowing better resolution of images. The examiner scans the nerve in the retrobulbar area and shifts the beam to display the 'intramural' segment of the nerve where a cup is to be expected. An initial, semi-quantitative impression of the size of the cup was made during the dynamic scanning; this was recorded as 'small', 'medium' or 'large'. Best images were frozen and polaroid photographs were taken for subsequent measurements of the disc cup margins with callipers.

The retrobulbar optic nerve was measured with standardised A-scan as previously described. ${ }^{10}$ Briefly, the instrument was set at T-sensitivity and the probe placed on the sclera at the temporal equator, aiming nasally and posteriorly. Fine adjustment of the probe was made while the patient fixated gaze at the primary position or slightly infero- 
temporally. Three optimal traces of the optic nerve were chosen and measurements were taken sheath to sheath with electronic callipers; the results were averaged.

\section{RESULTS}

There were 11 men and 14 women. Of these 6 were normals, 1 had low tension glaucoma, 4 were ocular hypertensives, 1 had optic nerve hypoplasia and 13 had chronic simple glaucoma. The average age was 65 years (range 20-91 years). The average optical disc diameter was $1.9 \pm 0.3 \mathrm{~mm}$ and the $\mathrm{C}: \mathrm{D}$ ratio was 0.6 (range $0-0.9$ ). The average retrobulbar nerve width was $2.88 \mathrm{~mm}$ (range $2.5-3.72 \mathrm{~mm}$ ). The optical cup diameter was then plotted against the ultrasonic cup diameter, as shown in Fig. 2. Fig. 3 shows the ultrasonic cup diameter plotted against the C:D ratio. Some of the points on the graph represent multiple data pairs.

Both optical cup diameter and $\mathrm{C}: \mathrm{D}$ ratio on linear regression correlate highly with the ultrasonic measurement. The two-tailed $p$ value is $<0.0001$ and considered extremely significant. There is no correlation between optic disc diameter and retrobulbar optic nerve diameter (Fig. 4).

Three discs with large C:D ratios and small vertical disc diameters were reported as small on echography. Two discs with no appreciable cup optically were identified correctly on ultrasound. All large cups were identified as large. These examples are shown in Figs. 5-7 illustrating different size cups on echography. A right eye shows a small cup (Fig. 5a); this is difficult to identify on ultrasonography but can be identified (Fig. 5b). A left eye (Fig. 6a) has a significant cup which is identifiable on ultrasonography (Fig. 6b). Very large cups can easily be identified (Fig. 7).

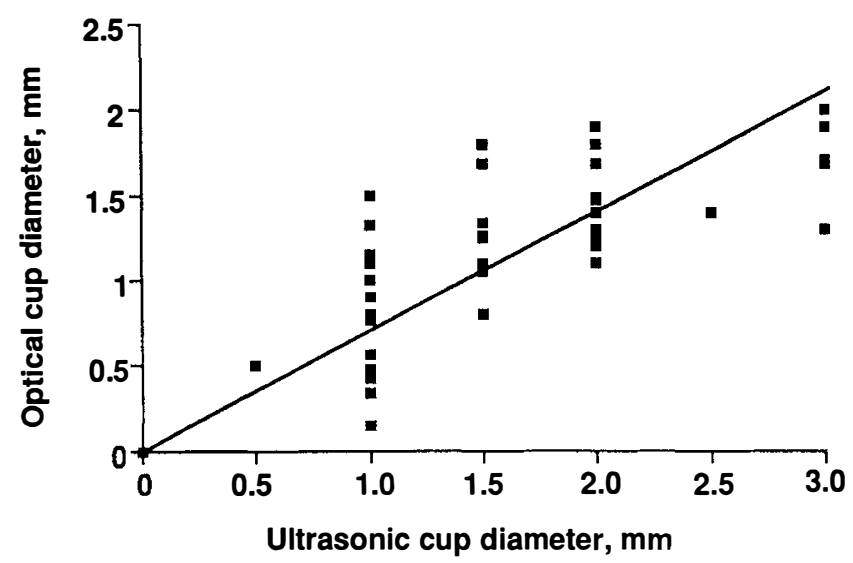

Optical cup size $-Y=(1.34) X+0.00$

Fig. 2. Optical cup diameter versus ultrasonic cup diameter.

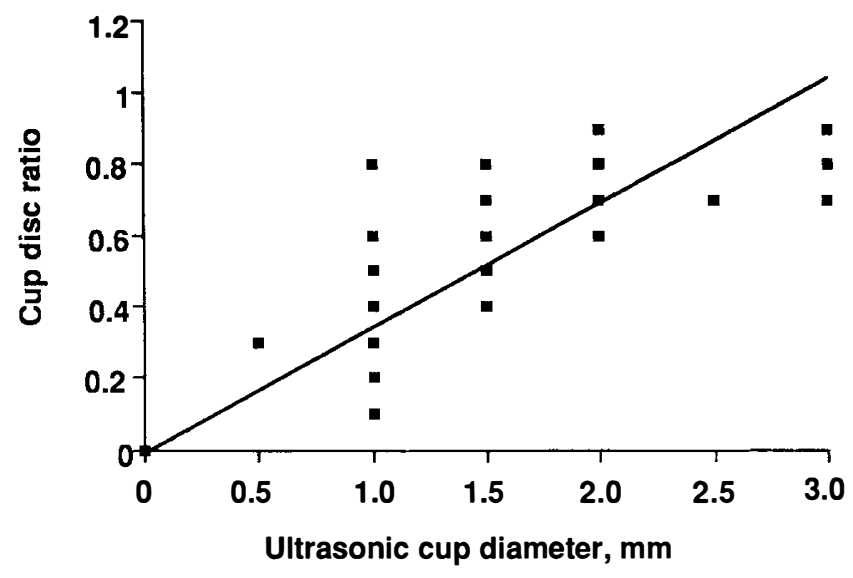

Disc Ratio $-Y=(1.34) X+0.00$

Fig. 3. Ultrasonic cup diameter versus cup disc ratio.

\section{DISCUSSION}

There is a normal variation in the diameter of the optic disc. ${ }^{5-7}$ This in turn leads to a non-pathological variation in the optic disc cup. ${ }^{8}$ A larger disc has a larger cup and a smaller disc may have little or no cup. When the optic disc is damaged by glaucoma there is an increase in the optic disc cup and thus a change in the $\mathrm{C}: \mathrm{D}$ ratio. ${ }^{11} \mathrm{~A}$ small disc may therefore be more damaged for a given $\mathrm{C}: \mathrm{D}$ ratio. ${ }^{12}$ This causes problems in clinical evaluation of the disc, and disc size is part of the routine assessment of glaucoma suspects. ${ }^{13}$ In ultrasonographic assessment of the disc the echographer is severely disadvantaged as there is no echographic feature corresponding to the disc edge; thus only the diameter and depth of the cup itself can be assessed.

There are many methods of measuring the optic disc. Clinically direct and indirect methods can be used. Studies with the Volk $90 \mathrm{D}$ lens have shown a good correlation with photographic assessment. A

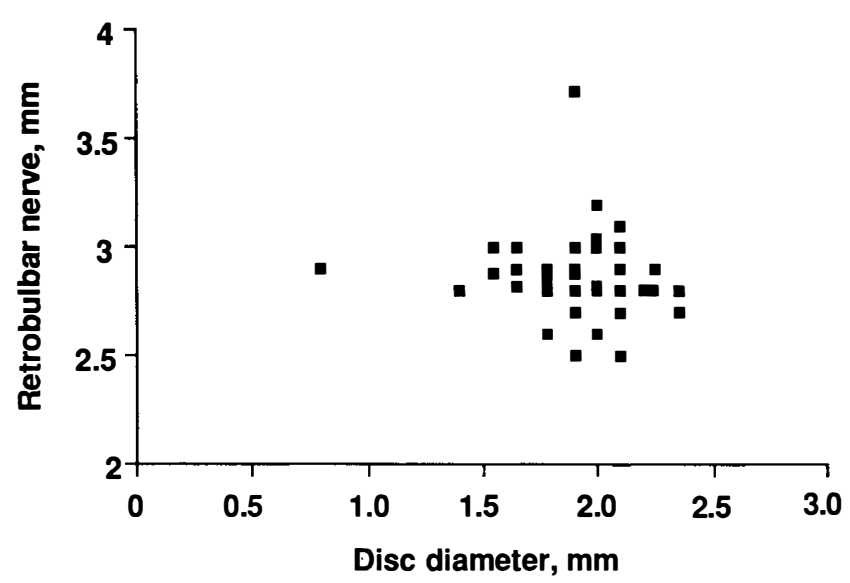

Retrobulbar nerve diameter

Fig. 4. Optic disc diameter versus retrobulbar optic nerve diameter. 


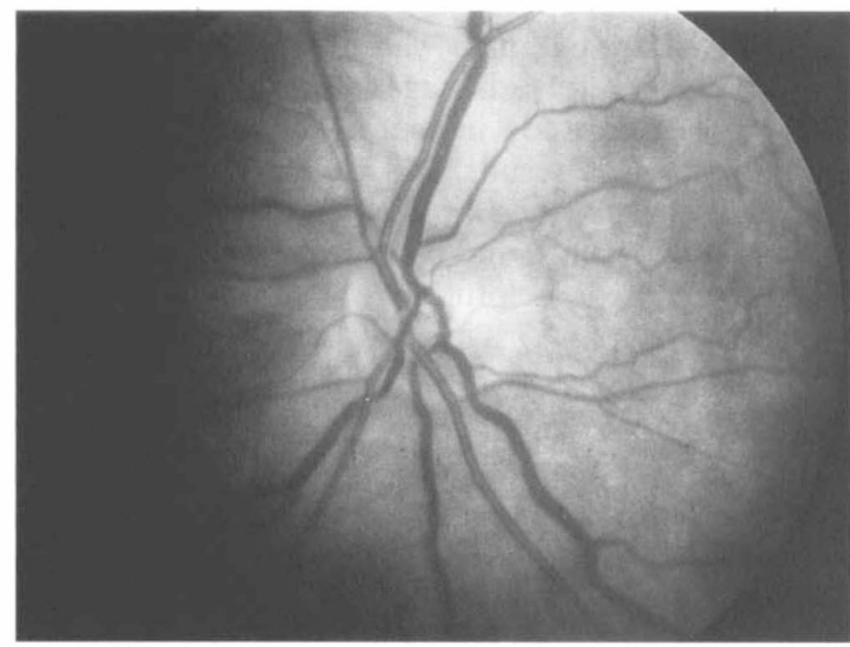

(a)

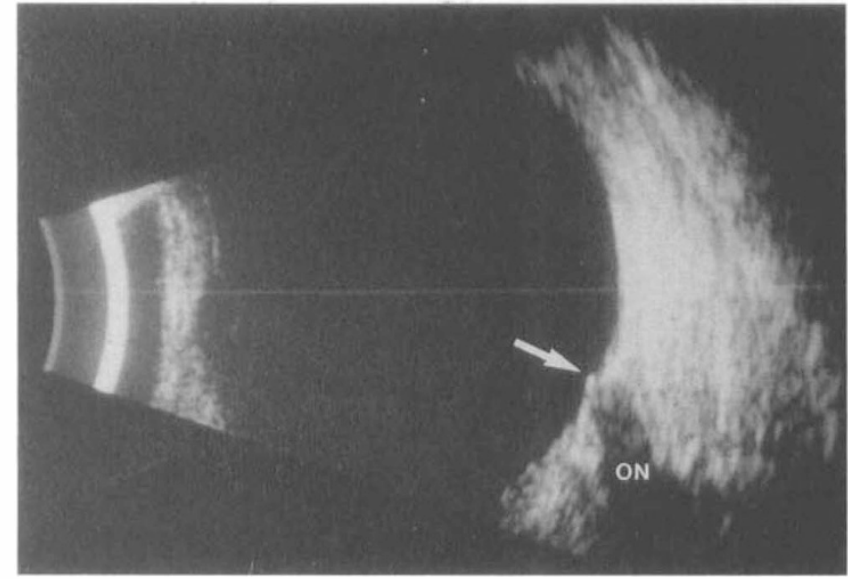

(b)

Fig. 5. A picture (a) and an ultrasound scan (b) of a small cup. The arrow shows the position of the optic nerve head; a small cup is just visible. The retrobulbar optic nerve is shown $(\mathrm{ON})$.

recent study has highlighted the errors of the $78 \mathrm{D}$ lens. ${ }^{14}$ The Zeiss four-mirror lens has also been used with reliable results. ${ }^{13}$ Photographic measurements are useful in research and seem to give reliable results by using corrective formulae; ${ }^{15.16}$ these, however, rely heavily on experience and equipment. All these measures seek an absolute measurement of optic disc diameter. Our optical examination was intended to identify small and large discs; we therefore were not attempting to measure cup diameter accurately with B-scan ultrasound as at present this technology is incapable of accurate measurement. Increasing resolution requires increasing frequency of ultrasound so there is less penetration, as in the high-frequency ultrasonic biomicroscope. We were already aware from previous studies that the scanner could only detect a

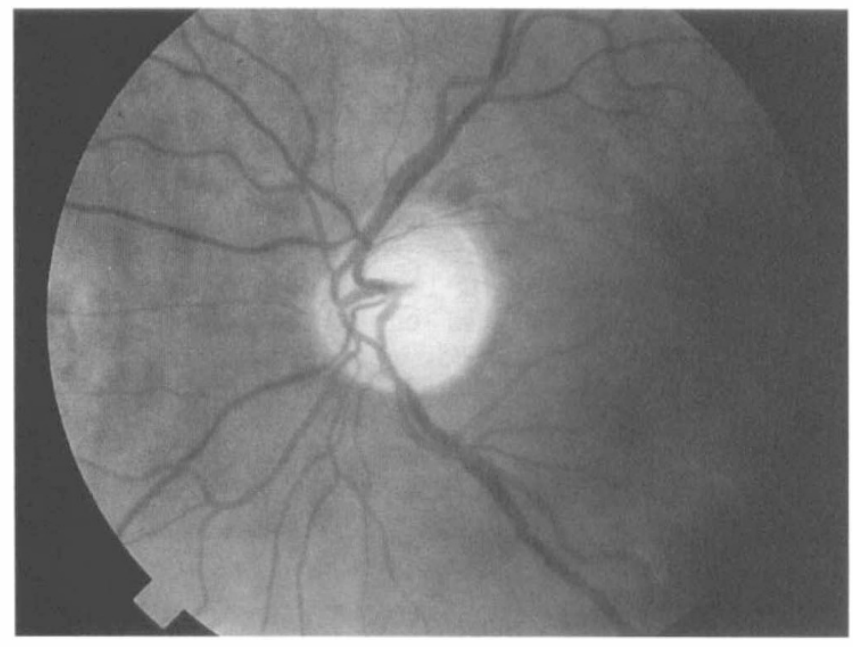

(a)
$\mathrm{C}: \mathrm{D}$ ratio of 0.6 or more. Assuming previous studies were of patients with fairly normal-sized discs (as the optical disc diameter was not quoted) this represents a resolution of about $0.8 \mathrm{~mm}$. Thus at this resolution the errors of the $90 \mathrm{D}$ lens would be insignificant for the purpose of this study. In our study the slit lamp was first calibrated, and patients were selected from the general clinics specifically for emmetropia, as it has been shown that ametropia contributes to errors in these methods. ${ }^{14}$

All eyes were used in the study as it was a test of the ability of ultrasound to detect the various sizes of cups. Some patients had different disc cups in the two eyes and thus were a good test of ultrasound's ability. The spread of our results confirms previous research ${ }^{14}$ in that there are errors of agreement in these methods.

Our study shows that there is a role for modern eye-dedicated ultrasound scanners in the detection of

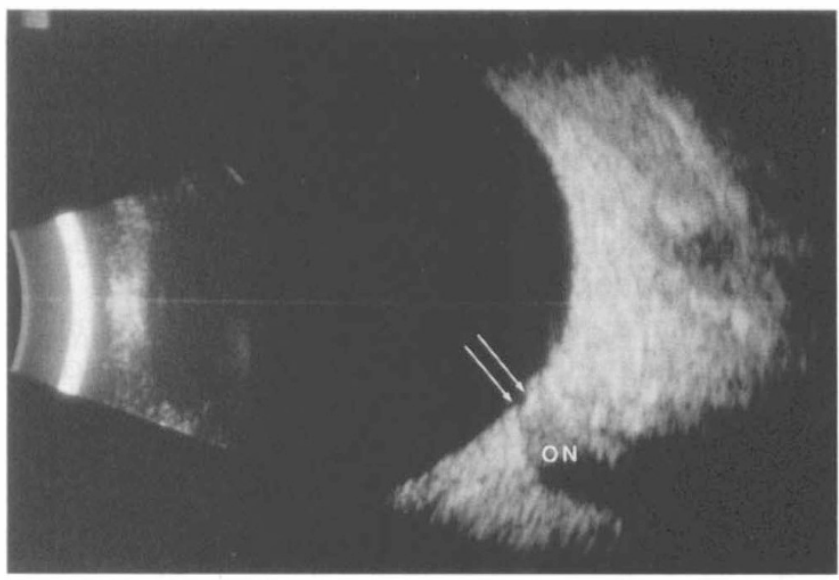

(b)

Fig. 6. A picture (a) and an ultrasound scan (b) of a large cup. The arrows show the edges of the disc. The edge is identified as the point where the posterior interface changes direction from the normal contour of the globe. The retrobulbar optic nerve is shown $(\mathrm{ON})$. 


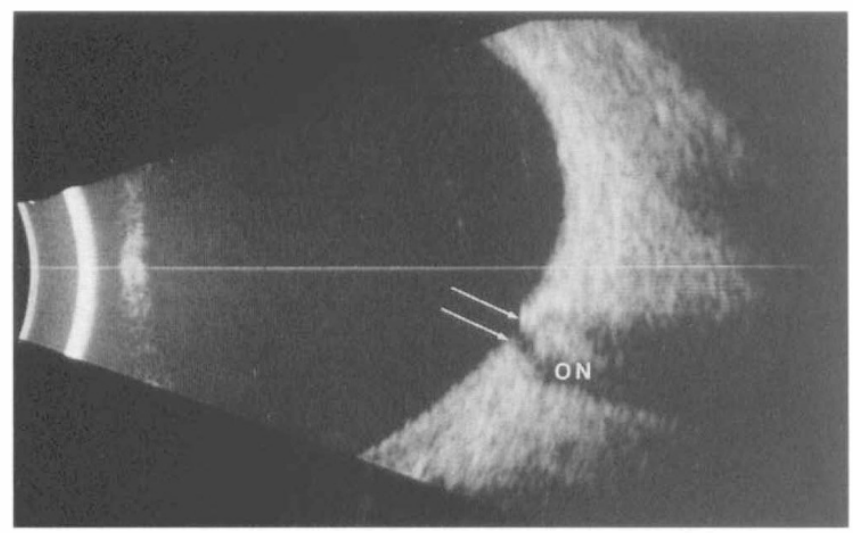

Fig. 7. An ultrasound scan of a very large cup. The arrows show the edges of the disc. The retrobulbar optic nerve is shown (ON).

optic disc cupping. This could be useful in screening patients with dense cataract, particularly those with a history of glaucoma. Sensitivity of ultrasonic optic disc cup detection depends on the type of instrument, methods of examination and operator skill. From our experience the best images of the disc cup are achieved when scanning is performed directly on the sclera. This tends to improve resolution and allows the patient to direct gaze towards the desired position more easily. Better images are also obtained when the ultrasound beam avoids the lens. In the case of the optic nerve head the best sections are transverse (marker up) and longitudinal (marker at the cornea) with the probe placed on the temporal sclera (Fig. 1). We realise that these sections may be off axis or oblique. These measurements therefore do not represent true anatomical dimensions of the disc or the nerve. Nevertheless they are consistent (same instrument, examination technique and examiner) and meaningful in the context of a study which aims to test the ability of echography to detect disc cups and correlate with an optical measure of optic disc diameter.

Our data suggest that a high-resolution contact Bultrasound scanner can detect vertical optic disc cups of $0.5 \mathrm{~mm}$ or larger. In addition a high corelation was found between echographic measurements of optic disc cups and optical vertical cup diameters and C:D ratios. However, three discs with $\mathrm{C}: \mathrm{D}$ ratios of 0.7 or larger were identified as small on ultrasound as they were in small-diameter optic discs. We therefore conclude that the B-scanner is measuring absolute cup size and that referring to the $\mathrm{C}: \mathrm{D}$ ratio in ultrasound may be misleading. ${ }^{1-4}$ Also, diameter of the retrobulbar optic nerve size did not show a correlation with disc size. In patients with obscure media B-scanning may well be a useful tool in identifying severely damaged discs. However, the findings of this study indicate caution in the interpretation of such results, and the clinician must take disc size into account. In patients with obscure media examination visually and by B-scanning of the fellow optic disc may be helpful.

\section{REFERENCES}

1. Darnley-Fisch D, Frazier Byrne S, Hughes JR, Parrish RK, Feuer MS. Contact B-scan echography in the assessment of optic nerve cupping. Am J Ophthalmol 1990;109:55-61.

2. Skalka HW. Ultrasonography of the optic nerve. In: Smith JL, editor. Neuroophthalmology update. New York: Masson, 1977:119-30.

3. Oissoinig KC. Standardised echography: basic principles, clinical applications and results. Int Ophthalmol Clin 1919;19:127.

4. Cohen JS, Stone RD, Hetherington J, Bullock J. Glaucomatous cupping of the optic disc by ultrasonography. Am J Ophthalmol 1976;82:24.

5. Bengtsson $B$. The variation and covariation of cup and disc diameters. Acta Ophthalmol (Copenh) 1976; $54: 804$

6. Caprioli J, Miller JM. Optic disc rim area is related to disc size in normal subjects. Arch Ophthalmol 1987;105:1683-5.

7. Jonas JB, Gusek GC, Guggenmoos-Holzmann I, Nauman GOH. Variability of the real dimensions of normal human optic discs. Graefes Arch Clin Exp Ophthalmol 1988;226:332-6.

8. Quigley HA, Brown AE, Morrison JD, Drance SM. The size and shape of the optic disc in the normal human eye. Arch Ophthalmol 1990;108:51-7.

9. Rubin S. Estimation of optic disc size using indirect biomicroscopy. Br J Ophthalmol 1994;78:363-5.

10. Atta HR. Imaging of the optic nerve with standardised echography. Eye 1988;2:358-66.

11. Tuuonen A, Airaksinen PJ. Initial glaucomatous optic disc and retinal nerve fibre layer abnormalities and their progression. Am J Ophthalmol 1991;111:485-90.

12. Hejl A, Molder H. Optic disc diameter influences the ability to detect glaucomatous optic disc damage. Arch Ophthalmol (Copenh) 1993;71:122-9.

13. Spencer AF, Vernon SA. Optic disc measurement with the Zeiss 4 mirror contact lens. $\mathrm{Br} \mathrm{J}$ Ophthalmol 1994;78:775-80.

14. Spencer AF, Vernon SA. Optic disc measurement: a comparison of indirect methods. $\mathrm{Br} \mathrm{J}$ Ophthalmol 1995;79:910-5.

15. Takamoto T, Schwartz B. Reproducibility of photogrammetric optic disc cup measurements. Invest Ophthalmol Vis Sci 1985;26:814-7.

16. Quigley HA, Dunkelberger GR, Green WR. Retinal ganglion cell atrophy correlated with automated perimetry in human eyes with glaucoma. Am J Ophthalmol 1989;107:453-64.

17. Bengtson B, Krakau CET. Correction of optic disc measurements on fundus photographs. Graefes Arch Clin Exp Ophthalmol 1992;230:24-8. 